Supporting Information

\title{
An Iridium(III) Complex that Exhibits Dual Mechanism Non-Linear Absorption
}

\author{
Kye-Young Kim, Richard T. Farley and Kirk S. Schanze* \\ Department of Chemistry, University of Florida P.O. Box 117200 \\ Gainesville, FL, 32611-7200, USA
}

*Author to whom correspondence should be addressed.

TEL: $352-392-9133$

FAX: $352-392-2395$

e-mail: kschanze@chem.ufl.edu

http://www.chem.ufl.edu/ kschanze/ 


\section{Experimental Section}

General Methods for Synthesis. All starting materials were used without further purification unless otherwise noted. All reactions were carried out under argon atmosphere with freshly distilled solvents. Compounds $\mathbf{A}$ and $\mathbf{B}$ were synthesized according to literature procedure. ${ }^{1,2}$ Column chromatography was performed on silica gel (Silycycle). ${ }^{1} \mathrm{H}$ and ${ }^{13} \mathrm{C}$ NMR spectra were recorded on a Varian Mercury 300 Spectrometer; Chemical shift was reported in ppm relative to TMS or residual protiated solvent signal. Elemental analysis was performed by University of Florida Spectroscopic Services.

Scheme S1. Synthetic schemes for ML and L
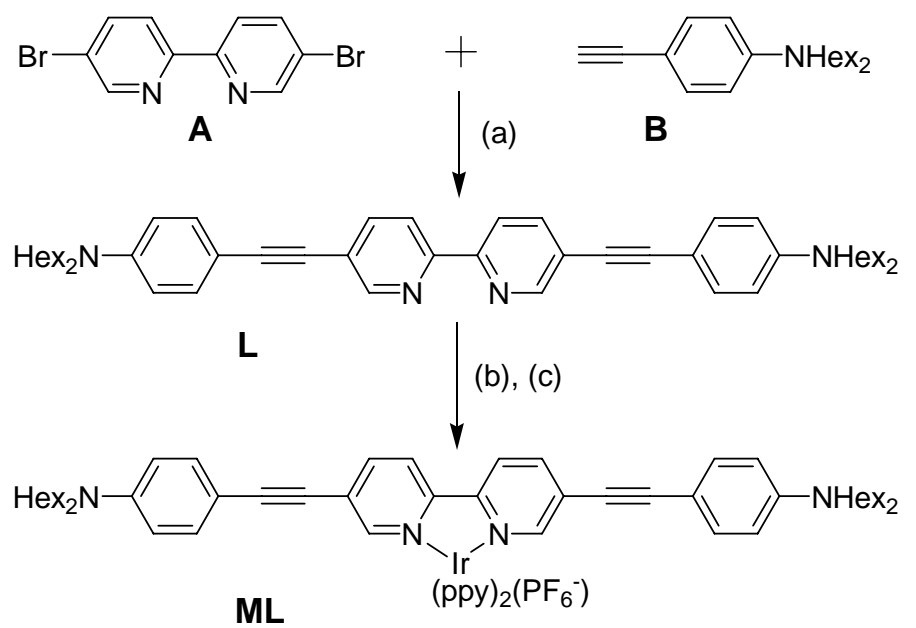

(a) $\mathrm{Pd}\left(\mathrm{PPh}_{3}\right)_{4}, \mathrm{Cul}, \mathrm{iPr}_{2} \mathrm{NH}, \mathrm{DMF}, 60^{\circ} \mathrm{C}, 73 \%$; (b) $\left(\operatorname{Ir}(\mathrm{ppy})_{2} \mathrm{Cl}\right)_{2}$, MeOCH $\mathrm{CH}_{2} \mathrm{OH}$, reflux; (c) $\mathrm{NH}_{4} \mathrm{PF}_{6}, 57 \%$

Ligand L. A stirred solution of 5,5'-dibromo-2,2'-bipyridine A (0.38 g, $1.2 \mathrm{mmol})$ and N,N-dihexyl-4-aminophenylethynyl B (0.69 g, $2.4 \mathrm{mmol}), \mathrm{Pd}\left(\mathrm{PPh}_{3}\right)_{4}(55 \mathrm{mg}, 48 \mathrm{~mol})$ and $\mathrm{CuI}$ (9.1 mg, $48 \mathrm{~mol})$ in $\mathrm{iPr}_{2} \mathrm{NH} / \mathrm{DMF}(20 \mathrm{~mL}, 1: 1 \mathrm{v}: \mathrm{v})$ was deoxygenated with argon for $30 \mathrm{~min}$. 
The mixture was stirred at $60{ }^{\circ} \mathrm{C}$ for $12 \mathrm{~h}$. The solvent was removed under the reduced pressure until dark brown slurry formed. The slurry was redissolved in $\mathrm{CH}_{2} \mathrm{Cl}_{2}(30 \mathrm{~mL})$ and the resulting solution was washed with water $(30 \mathrm{~mL} \times 2)$, dried over $\mathrm{MgSO}_{4}$ and rotavaped to give a brown solid. Column chromatography on silica gel (ethyl acetate/hexane, 1/7) gave a crude product as a reddish brown solid. Recrystallization from $\mathrm{CH}_{2} \mathrm{Cl}_{2} /$ hexane at $-10{ }^{\circ} \mathrm{C}$ yielded ligand $\mathbf{L}$ as a yellow powder $(0.75 \mathrm{~g}, 73 \%):{ }^{1} \mathrm{H} \mathrm{NMR}\left(300 \mathrm{MHz}, \mathrm{CDCl}_{3}\right) \delta 8.76(\mathrm{~d}, J=2.0 \mathrm{~Hz}$, 2H), $8.36(\mathrm{~d}, J=8.5 \mathrm{~Hz}, 2 \mathrm{H}), 7.88(\mathrm{dd}, J=8.5$ and $2.0 \mathrm{~Hz}, 2 \mathrm{H}), 7.40(\mathrm{~d}, J=9.1 \mathrm{~Hz}, 4 \mathrm{H}), 6.58$ (d, $J=9.1 \mathrm{~Hz}, 4 \mathrm{H}), 3.29(\mathrm{t}, J=7.6 \mathrm{~Hz}, 8 \mathrm{H}), 1.59(\mathrm{~m}, 8 \mathrm{H}), 1.40-125(\mathrm{~m}, 24 \mathrm{H}), 0.91(\mathrm{t}, J=6.5$ $\mathrm{Hz}, 12 \mathrm{H}) ;{ }^{13} \mathrm{C}$ NMR $\left(75 \mathrm{MHz}, \mathrm{CDCl}_{3}\right) \delta 153.3,151.3,148.2,138.7,133.0,121.3,120.3,111.1$, 107.7, 95.6, 84.5, 50.9, 31.7, 27.2, 26.8, 22.7, 14.1; Anal. cald. for $\mathrm{C}_{50} \mathrm{H}_{66} \mathrm{~N}_{4}(\%)$ : C, 83.05; $\mathrm{H}$, 9.20; N, 7.75; found C, 83.22; H, 9.46; N, 7.49.

Ir(III) complex ML. A suspension of ligand $\mathbf{L}(0.270 \mathrm{~g}, 0.374 \mathrm{mmol})$ and $\left[\operatorname{Ir}(\mathrm{ppy})_{2} \mathrm{Cl}\right]_{2}{ }^{3}$ $(0.200 \mathrm{~g}, 0.187 \mathrm{mmol})$ in 2-methoxyethanol $(15 \mathrm{~mL})$ was stirred at reflux for $24 \mathrm{hrs}$. The solvent was then reduced to $\sim 5 \mathrm{~mL}$ in vacuo and poured into a saturated aqueous solution of $\mathrm{NH}_{4} \mathrm{PF}_{6}$. The resulting black precipitate was collected by suction filtration, dried under vacuum and purified by column chromatography on silica gel. Elution with ethyl acetate/hexane (1/7) removed the residual ligand $\mathbf{L}$, and further elution with ethyl acetate $/ \mathrm{CH}_{2} \mathrm{Cl}_{2}(1 / 3)$ gave the $\mathrm{Ir}$ complex ML as a reddish black solid. (0.268 g, 57\%): ${ }^{1} \mathrm{H}$ NMR (300 MHz, acetone-d $\left.{ }_{6}\right) \delta 8.76$ $(\mathrm{d}, J=8.5 \mathrm{~Hz}, 2 \mathrm{H}), 8.25(\mathrm{~d}, J=9.1 \mathrm{~Hz}, 2 \mathrm{H}), 8.20(\mathrm{dd}, J=6.7$ and $2.0 \mathrm{~Hz}, 2 \mathrm{H}), 8.10-7.95(\mathrm{~m}$, $6 \mathrm{H}), 7.92(\mathrm{~d}, J=7.9 \mathrm{~Hz}, 2 \mathrm{H}), 7.25-7.16(\mathrm{~m}, 6 \mathrm{H}), 7.06(\mathrm{td}, J=7.3$ and $1.2 \mathrm{~Hz}, 2 \mathrm{H}), 6.97(\mathrm{td}, J=$ 7.3 and $1.2 \mathrm{~Hz}, 2 \mathrm{H}), 6.68(\mathrm{~d}, J=9.1 \mathrm{~Hz}, 4 \mathrm{H}), 6.36(\mathrm{~d}, J=7.3 \mathrm{~Hz}, 2 \mathrm{H}), 3.37(\mathrm{t}, J=7.6 \mathrm{~Hz}, 8 \mathrm{H})$, $1.59(\mathrm{~m}, 8 \mathrm{H}), 1.37-1.27(\mathrm{~m}, 24 \mathrm{H}), 0.85(\mathrm{t}, 12 \mathrm{H}) ;{ }^{13} \mathrm{C} \mathrm{NMR}\left(75 \mathrm{MHz}, \mathrm{CDCl}_{3}\right) \delta 168.4,153.9$, $152.3,150.7,150.5,150.1,144.9,141.0,139.6,134.1,132.4,131.3,126.4,125.9,125.4,124.6$, 
$123.5,120.8,112.2,106.7,101.1,83.8,51.3,32.4,27.8,27.3,23.3,14.3$; Anal. cald. for $\mathrm{C}_{72} \mathrm{H}_{82} \mathrm{~F}_{6} \mathrm{IrN}_{6} \mathrm{P}(\%)$ : C, 63.18; H, 6.04; N, 6.14; found C, 63.42; H, 6.41; N 5.68; HRMS (ESIFTCR-MS) cald. [(M-PF $\left.\left.{ }_{6}\right)+\mathrm{H}\right] ; 1221.6160$; found $\left[\left(\mathrm{M}^{-} \mathrm{PF}_{6}\right)+\mathrm{H}\right] 1221.6201$

Photophysical Measurements. All photophysical studies were carried out in 1 x $1 \mathrm{~cm}$ quartz cuvettes, unless otherwise noted. All sample solutions were prepared in dry THF and deoxygenated by using argon bubbling unless otherwise noted. UV-visible absorption spectra were recorded on a Varian Cary 100 dual-beam spectrophotometer. For emission measurements, sample concentrations were adjusted to produce optically dilute solutions $\left(\mathrm{A}_{\max }<\right.$ 0.2). Transient absorption measurements were carried out with solutions having $\mathrm{A}=0.8-1.0$ at $355 \mathrm{~nm}$. Corrected steady-state emission measurements were performed on a SPEX F-112 fluorescence spectrometer equipped with Hamamatsu R928 PMT for visible measurement. Emission quantum yields were measured relative to $\mathrm{Ru}(\mathrm{bpy})_{3} \mathrm{Cl}_{2}$ in air saturated $\mathrm{H}_{2} \mathrm{O}$ where $\phi=$ 0.0379. ${ }^{4}$ Transient absorption spectra were obtained on an instrument that has been previously described that uses the third harmonic of a Nd:YAG laser (Spectra Physics GCR-14, $355 \mathrm{~nm}, 10$ ns fwhm, $10 \mathrm{~mJ}-$ pulse $^{-1}, 20 \mathrm{~mJ}-\mathrm{cm}^{-2}$ irradiance) as excitation source. ${ }^{5}$

Two-photon induced emission spectra were recorded on an apparatus which relies on a Continuum SureLite series Nd:YAG laser as an excitation source. The fundamental output of the laser $(\lambda=1064 \mathrm{~nm}, 10 \mathrm{~ns}$ fwhm) was passed through a telescope providing a final beam diameter $0.7 \mathrm{~cm}$. This beam was focused with a $10 \mathrm{~cm}$ focal length lens (Newport Optics) and the sample was positioned at the focal plane of the lens. The luminescence was detected by an intensified CCD detector (Princeton Instruments, PI-MAX iCCD) coupled to an Acton SpecraPro 150 spectrograph. The sample was measured in a $5 \mathrm{~mm}$ i.d. round borosilicate tube. Nonlinear transmittance was measured using the same apparatus, with samples contained in a 10 
mm pathlength quartz optical cell that was positioned at the focal plane. The energies of the incident and transmitted beams were measured with calibrated energy meters (Ophir analog power meter, and a Scientech P09 energy meter with a Vector S310 digital display, respectively).

Transient absorption arising from two-photon excitation was carried out using an unfocused beam from the Continuum SureLite laser, a Hamamatsu xenon flash lamp, and the previously mentioned spectrograph and CCD detector.

Fluence-dependent emission intensity arising from two-photon excitation was measured using a QuantaRay GCR Series Nd:YAG laser as the excitation source $(\lambda=1064,10 \mathrm{~ns}$ fwhm, unfocused $0.8 \mathrm{~cm}$ diameter beam) and a Hamamatsu R928 PMT coupled to an Oriel $1 / 8 \mathrm{~m}$ monochromator set at the emission $\lambda_{\max }$.

\section{References}

${ }^{1}$ Romero, F. M.; Ziessel, R. Tet. Lett. 1995, 36, 6471.

${ }^{2}$ Kapplinger, C.; Beckert, R. Synthesis. 2002, 13, 1843.

${ }^{3}$ Nonoyama, M. Bull. Chem. Soc. Jpn. 1974, 47, 767-768.

${ }^{4}$ Thornton, N, B. Chromophore Quenched Based Luminescence Probes for DNA; University of Florida: Gainesville, 1995.

${ }^{5}$ Wang, Y. S.; Schanze, K. S. Chem. Phys. 1993, 176, 305-319. 
Figure S-1. Two-photon transient absorption difference spectra of $5 \mathrm{mM}$ solution of ML in deoxygenated THF following $1064 \mathrm{~nm}$ pulsed excitation.

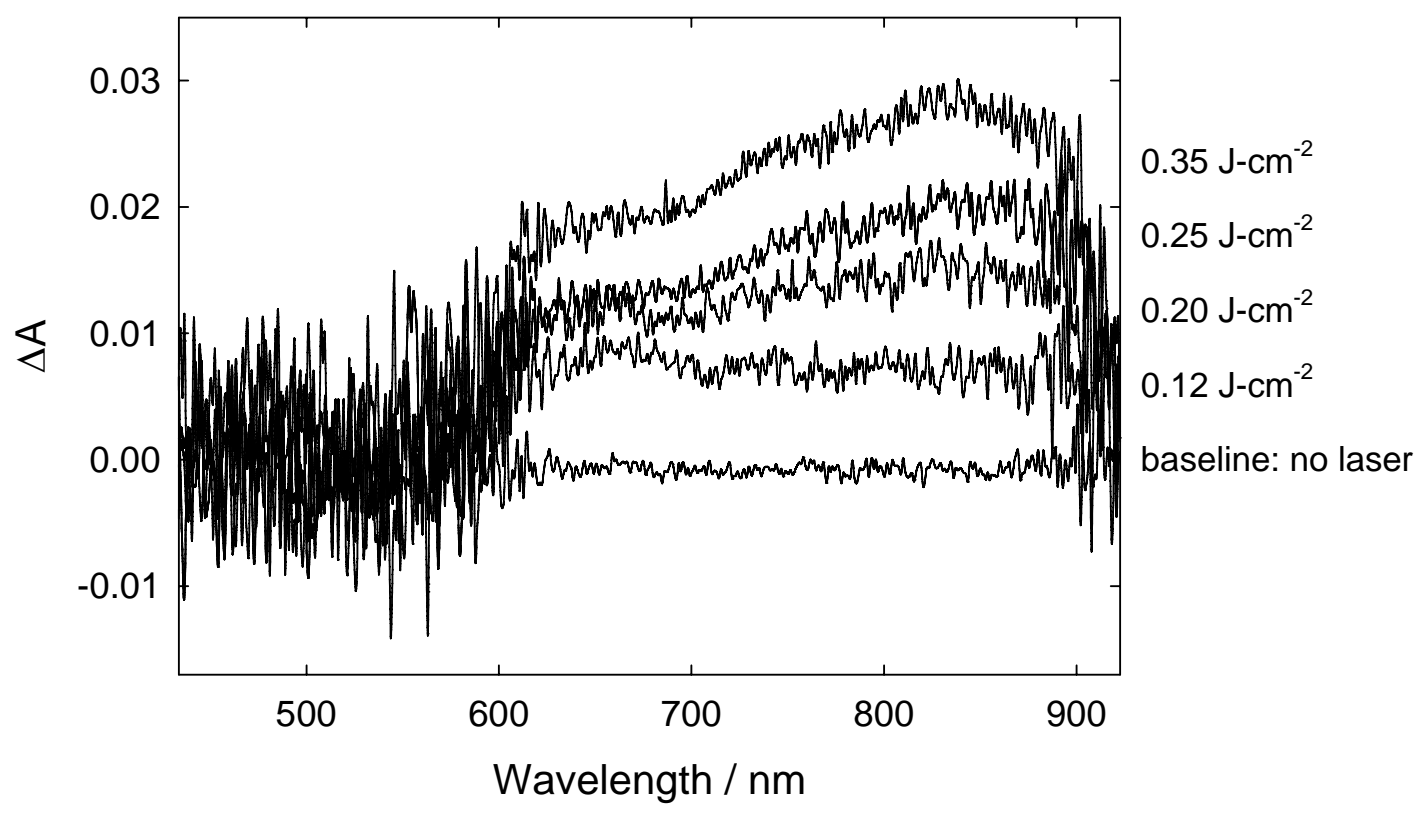


Figure S-2.

${ }^{1} \mathrm{H}$ NMR Spectrum of ML (300 MHz, acetone- $\mathrm{d}_{6}$ )

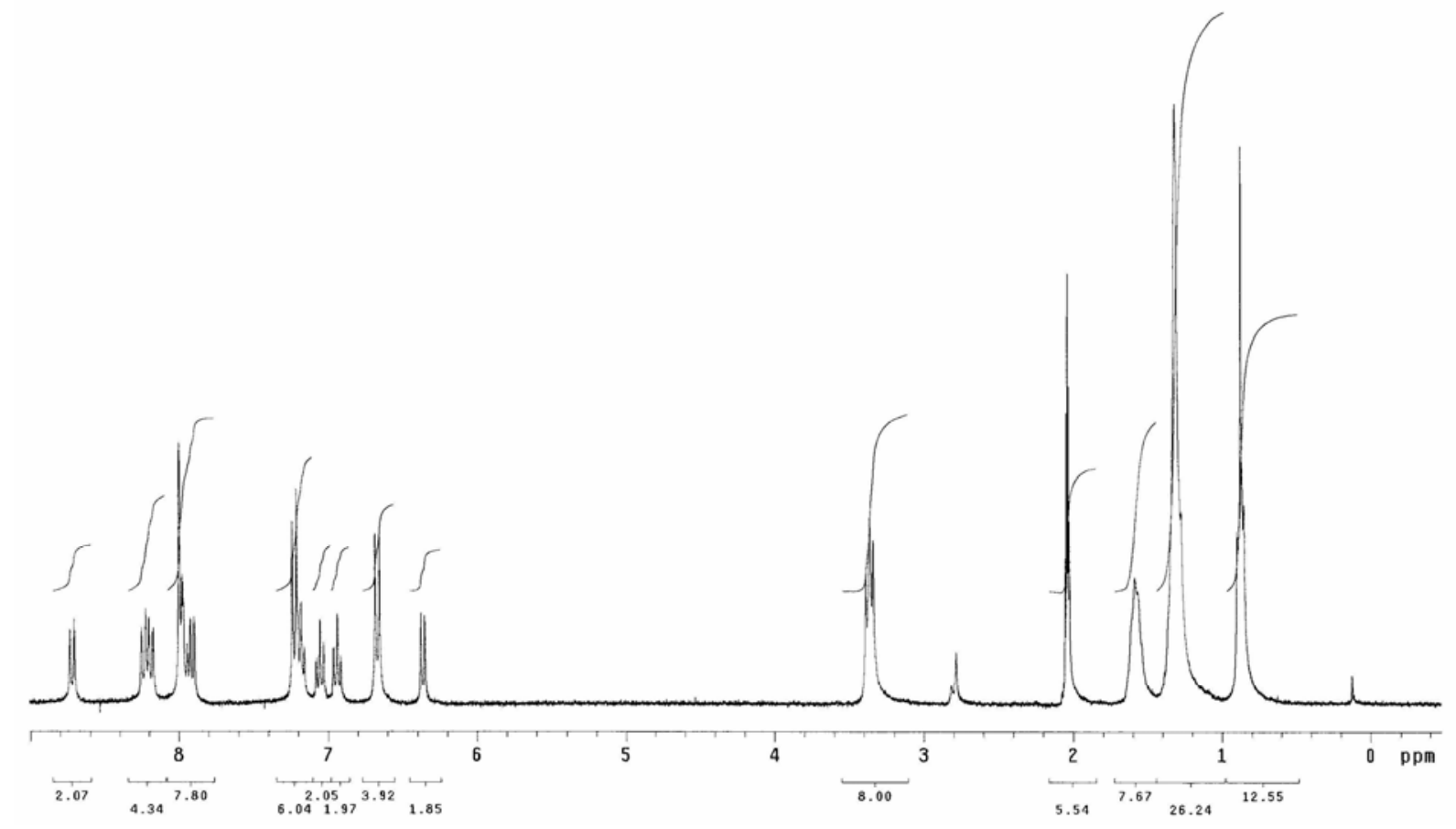

${ }^{13} \mathrm{C}$ NMR Spectrum of ML (75 MHz, acetone- $\mathrm{d}_{6}$ )

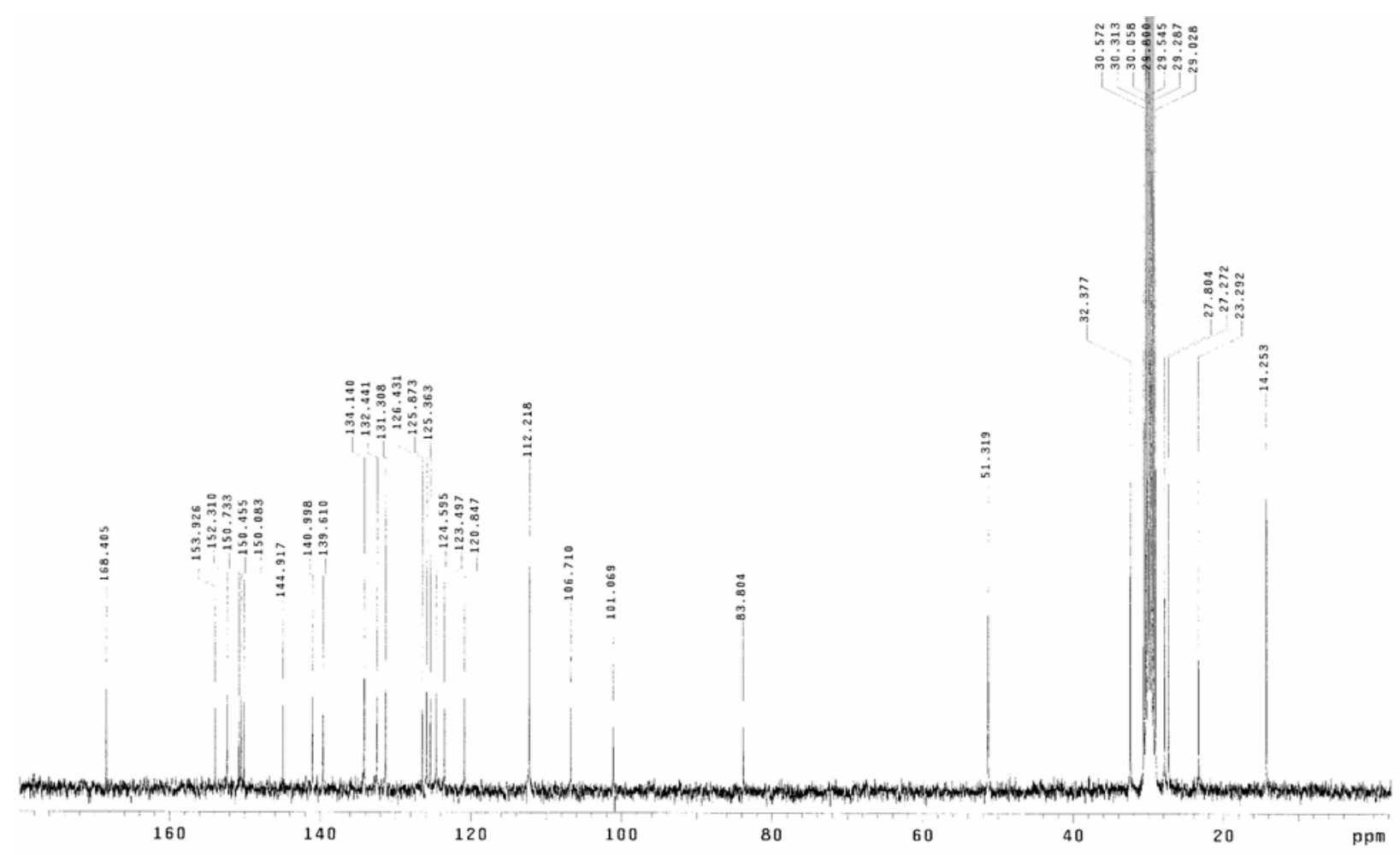


Figure S-3.

${ }^{1} \mathrm{H}$ NMR Spectrum of $\mathbf{L}\left(300 \mathrm{MHz}, \mathrm{CDCl}_{3}\right)$

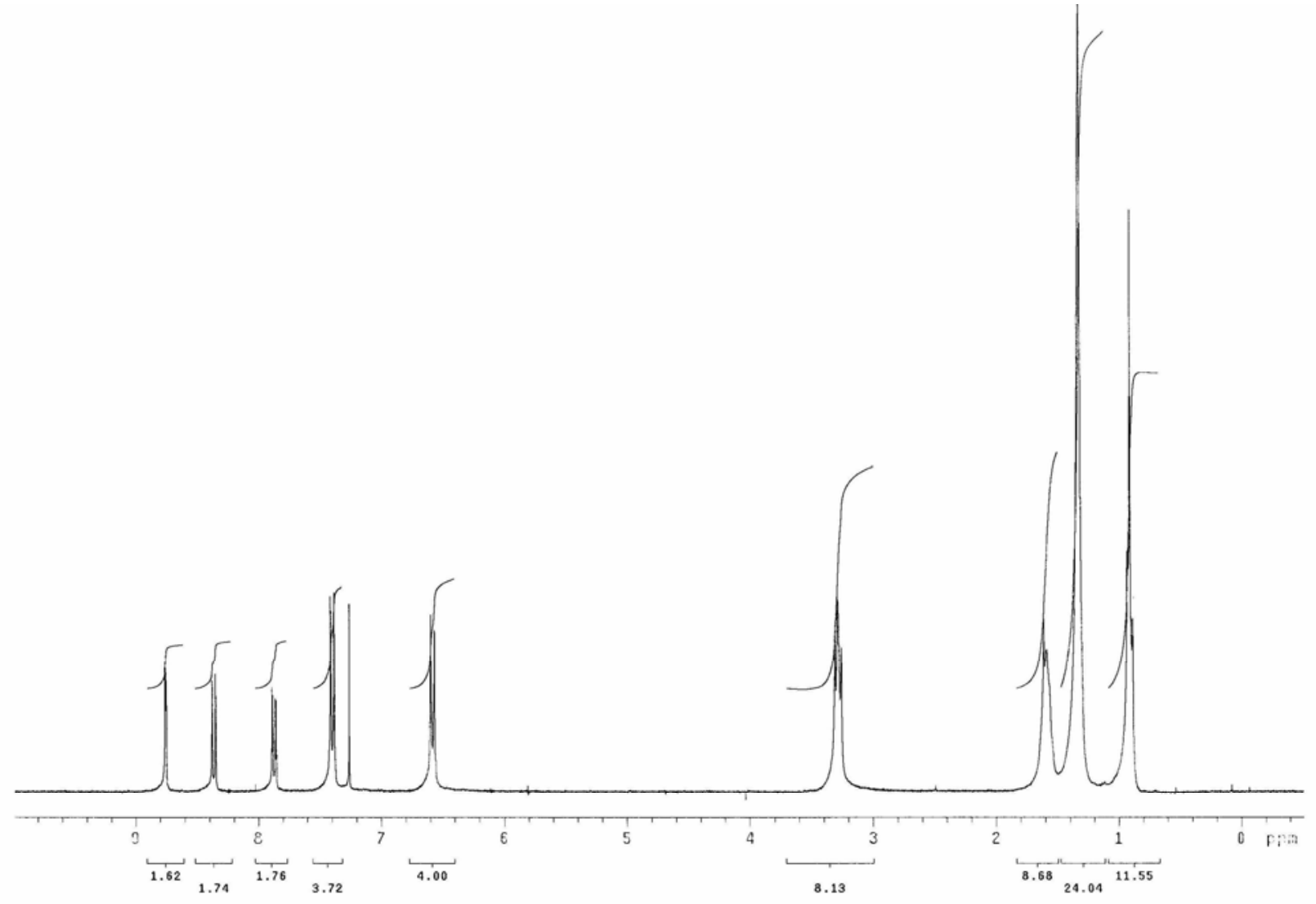

${ }^{13} \mathrm{C}$ NMR Spectrum of $\mathbf{L}\left(75 \mathrm{MHz}, \mathrm{CDCl}_{3}\right)$

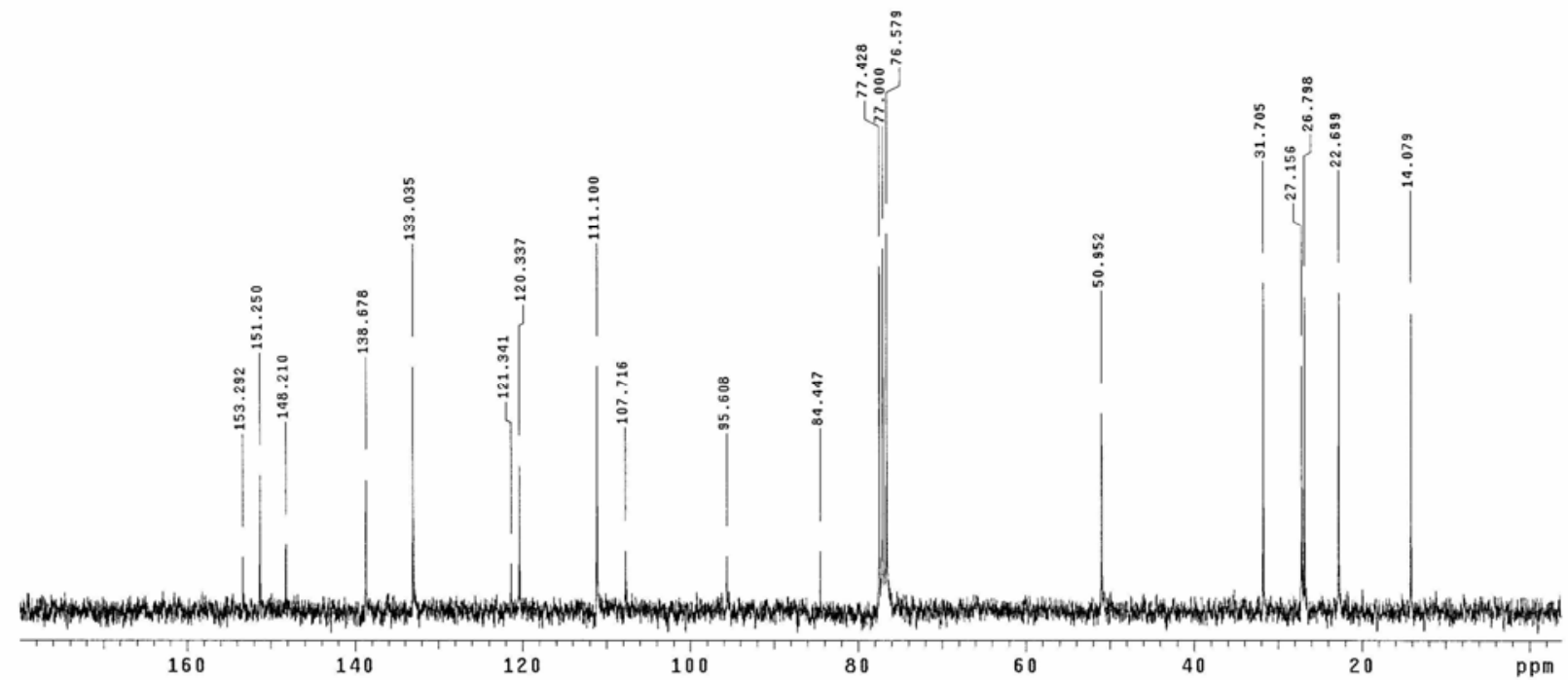

